\title{
Increased In Vivo Expression of an Inflammatory Marker in Temporal Lobe Epilepsy
}

\author{
Jussi Hirvonen ${ }^{1}$, William C. Kreisl ${ }^{1}$, Masahiro Fujita ${ }^{1}$ Irene Dustin $^{2}$, Omar Khan ${ }^{2}$, Shmuel Appel $^{2}$, Yi Zhang ${ }^{1}$, \\ Cheryl Morse ${ }^{1}$, Victor W. Pike ${ }^{1}$, Robert B. Innis ${ }^{1}$, and William H. Theodore ${ }^{2}$ \\ ${ }^{1}$ Molecular Imaging Branch, National Institute of Mental Health, National Institutes of Health, Bethesda, Maryland; and ${ }^{2}$ Clinical \\ Epilepsy Section, National Institute of Neurological Disorders and Stroke, National Institutes of Health, Bethesda, Maryland
}

\begin{abstract}
Animal studies and clinical observations suggest that epilepsy is associated with inflammation. Translocator protein (TSPO) (18 kDa), a marker of inflammation, is increased in vitro in surgical samples from patients with temporal lobe epilepsy. TSPO can be measured in the living human brain with PET and the novel radioligand ${ }^{11} \mathrm{C}-\mathrm{PBR} 28$. In this study, we sought to determine whether in vivo expression of TSPO is increased ipsilateral to the seizure focus in patients with temporal lobe epilepsy. Methods: Sixteen patients with unilateral temporal lobe epilepsy and 30 healthy subjects were studied with ${ }^{11} \mathrm{C}$ PBR28 PET and MRI. Uptake of radioactivity after injection of ${ }^{11} \mathrm{C}-\mathrm{PBR} 28$ was measured from regions of interest drawn bilaterally onto MR images. Brain uptake from ipsilateral and contralateral hemispheres was compared using a paired-samples $t$ test. Results: We found that brain uptake was higher ipsilateral to the seizure focus in the hippocampus, parahippocampal gyrus, amygdala, fusiform gyrus, and choroid plexus but not in other brain regions. This asymmetry was more pronounced in patients with hippocampal sclerosis than in those without. Conclusion: We found increased uptake of radioactivity after injection of ${ }^{11} \mathrm{C}$-PBR28 ipsilateral to the seizure focus in patients with temporal lobe epilepsy, suggesting increased expression of TSPO. Studies in larger samples are required to confirm this finding and determine the clinical utility of imaging TSPO in temporal lobe epilepsy.
\end{abstract}

Key Words: positron emission tomography; translocator protein $18 \mathrm{kDa}$; epilepsy; inflammation

J Nucl Med 2012; 53:234-240

DOI: 10.2967/jnumed.111.091694

$\mathbf{I}_{\mathrm{n}}$

fflammation may contribute to the pathophysiology of epilepsy. Animal studies have found that proinflammatory cytokines are increased in rodent models of epilepsy and that seizure frequency correlates with activation of these cytokines (1-3). Human studies have found increased expression of proinflammatory markers in neurosurgical sam-

Received Apr. 10, 2011; revision accepted Sep. 28, 2011

For correspondence or reprints contact: Robert B. Innis, Molecular Imaging Branch, National Institute of Mental Health, National Institutes of Health, 10 Center Dr., MSC-1026, Bldg. 10, Room B1D43, Bethesda, MD 20892-1026. E-mail: robert.innis@nih.gov

Published online Jan. 11, 2012.

COPYRIGHT @ 2012 by the Society of Nuclear Medicine, Inc. ples from patients with focal epilepsy $(2,4-6)$, and a recent metaanalysis suggests an association between epilepsy and a genetic functional polymorphism that causes increased production of the proinflammatory cytokine interleukin$1 \beta(7)$.

Translocator protein (TSPO) $(18 \mathrm{kDa})$ is a marker of inflammation $(8,9)$. Basal expression of TSPO is low, but in inflammation, activated microglia and astrocytes overexpress TSPO (10). TSPO expression is increased in vitro in surgical samples from patients with temporal lobe epilepsy $(11,12)$. In these samples, increased TSPO colocalizes with hippocampal sclerosis (12), a lesion associated with neuronal loss and glial proliferation.

TSPO expression has been measured in vivo with PET using the radioligand ${ }^{11} \mathrm{C}-(R)-\mathrm{PK} 11195$ (13). However, results from studies with ${ }^{11} \mathrm{C}-(R)$-PK11195 have not been consistent. Increased uptake of radioactivity after injection of ${ }^{11} \mathrm{C}-(R)$-PK11195 was reported in 2 cases of Rasmussen encephalitis (13) and 1 case of intractable seizures due to encephalopathy of unknown origin (14) but not in 3 stable patients with temporal lobe epilepsy and hippocampal sclerosis (13). Unfortunately, ${ }^{11} \mathrm{C}-(R)-\mathrm{PK} 11195$ has a low ratio of specific-to-nonspecific binding (15) and may therefore lack sufficient sensitivity to detect increased TSPO in temporal lobe epilepsy. We recently developed ${ }^{11} \mathrm{C}-\mathrm{PBR} 28$ as an improved PET radioligand for TSPO (16). ${ }^{11} \mathrm{C}-\mathrm{PBR} 28$ has a higher ratio of specific-to-nonspecific binding than ${ }^{11} \mathrm{C}-(R)$-PK11195 (15) and may have sufficient sensitivity to detect increased TSPO in temporal lobe epilepsy.

To determine whether ${ }^{11} \mathrm{C}$-PBR28 could detect increased TSPO, we scanned 16 patients with temporal lobe epilepsy. Because we expected increased TSPO to be associated with the seizure focus, we compared uptake in brain regions ipsilateral and contralateral to the focus in these patients as well as in right and left hemisphere regions in 30 healthy subjects.

\section{MATERIALS AND METHODS}

Subjects

Sixteen patients ( 8 men, 8 women; mean age \pm SD, $36 \pm 10$ y; mean body weight $\pm \mathrm{SD}, 72 \pm 15 \mathrm{~kg}$ ) with temporal lobe epilepsy were recruited by the Clinical Epilepsy Section at the National Institute of Neurologic Disorders and Stroke (Table 1). Clinical 


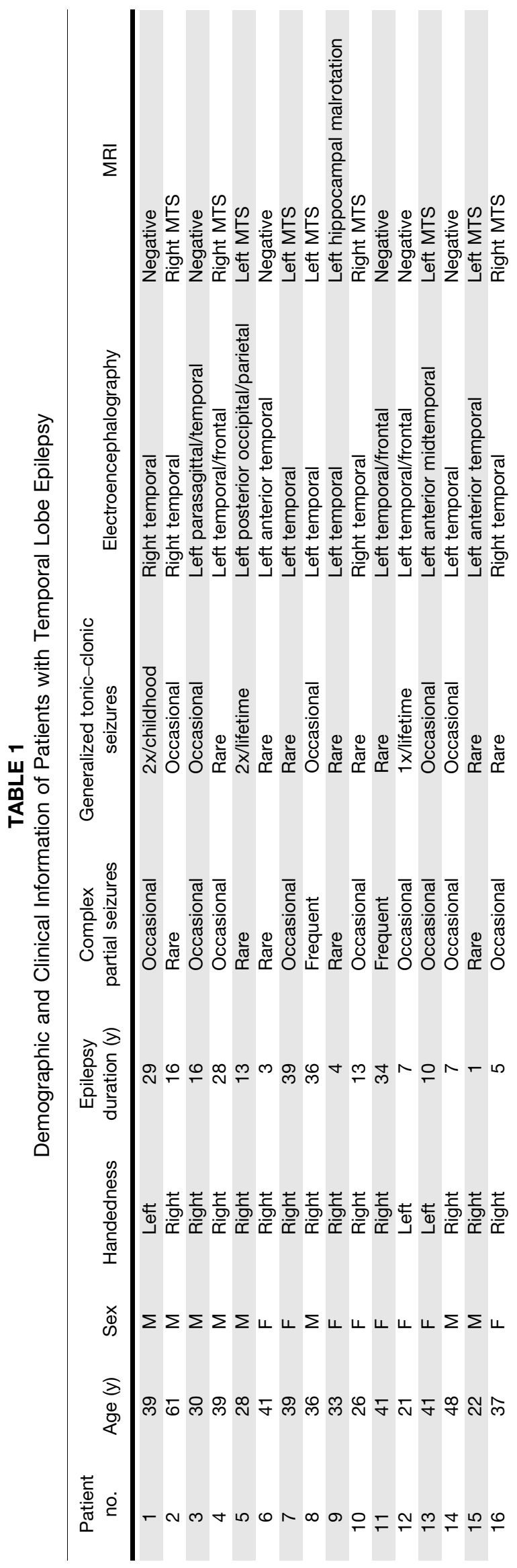

and diagnostic evaluation included detailed medical history, physical examination, laboratory tests of blood and urine, electrocardiogram, ictal videoelectroencephalography, and clinical MRI of the brain. Patients were excluded if they had serious medical conditions (other than epilepsy), had a history of alcohol or drug use disorder, or were pregnant. For each patient, seizure lateralization was based on the presence of unilateral hippocampal sclerosis on MRI or on the presence of lateralizing ictal electroencephalography findings if sclerosis was not present on MRI. In addition to the patients presented here, 1 female patient was recruited but had to be excluded because she had negligible binding of ${ }^{11} \mathrm{C}-\mathrm{PBR} 28$ to TSPO in the brain $(15,17)$ (see the "Discussion" section).

To examine physiologic asymmetry in ${ }^{11} \mathrm{C}$-PBR28 uptake, 2 cohorts of healthy subjects were analyzed: 7 healthy subjects were scanned with the High-Resolution Research Tomograph (HRRT) PET scanner (Siemens Medical Solutions) (4 men, 3 women; mean age $\pm \mathrm{SD}, 45 \pm 11 \mathrm{y}$; mean body weight $\pm \mathrm{SD}, 75 \pm 18$ $\mathrm{kg}$ ), and 23 subjects were scanned with the Advance PET scanner (GE Healthcare) (14 men, 9 women; mean age \pm SD, $48 \pm 17 \mathrm{y}$; mean body weight $\pm \mathrm{SD}, 78 \pm 14 \mathrm{~kg}$ ). Subjects underwent detailed medical history, physical examination, laboratory tests of blood and urine, and electrocardiogram for excluding serious medical conditions, illicit drug use, and pregnancy.

The subjects' consents were obtained according to the Declaration of Helsinki. The Institutional Review Board of the National Institute of Mental Health/National Institute of Neurological Disorders and Stroke approved the protocol and the consent forms.

\section{MRI}

All subjects underwent brain MRI as part of their evaluation. Healthy subjects underwent MRI either at $1.5 \mathrm{~T}$ or $3 \mathrm{~T}$ and patients with temporal lobe epilepsy at $3 \mathrm{~T}$. The MRI scans included clinical coronal and axial T1-weighed, T2-weighted, and fluid attenuation inversion recovery sequences. A high-resolution 3-dimensional T1-weighted sequence was obtained for anatomic reference for PET image analyses. The presence or absence of hippocampal sclerosis was determined by an experienced neuroradiologist.

\section{Preparation of ${ }^{11} \mathrm{C}$-PBR28}

${ }^{11} \mathrm{C}-\mathrm{PBR} 28$ was synthesized as previously described (16). Synthesis was performed in accordance with Investigational New Drug application 76,441. A copy of the application is available at http://pdsp.med.unc.edu/snidd/. ${ }^{11} \mathrm{C}-\mathrm{PBR} 28$ was synthesized with high radiochemical purity $(>99 \%)$ and had a specific activity at the time of injection of $126 \pm 77 \mathrm{GBq} / \mu \mathrm{mol}$ for patients with temporal lobe epilepsy, $64 \pm 30 \mathrm{GBq} / \mu \mathrm{mol}$ for healthy subjects scanned with the HRRT camera, and $114 \pm 68 \mathrm{GBq} / \mu \mathrm{mol}$ for healthy subjects scanned with the Advance camera.

\section{Image Acquisition}

After intravenous injection of ${ }^{11} \mathrm{C}-\mathrm{PBR} 28(684 \pm 48 \mathrm{MBq}$ for patients with temporal lobe epilepsy, $492 \pm 64 \mathrm{MBq}$ for healthy subjects scanned with the HRRT camera, and $637 \pm 82 \mathrm{MBq}$ for healthy subjects scanned with the Advance camera), PET images were acquired using the HRRT or the Advance as previously described (18) for $120 \mathrm{~min}$. All patients with temporal lobe epilepsy were scanned on the HRRT. We had a total of 7 healthy subjects scanned on the HRRT. To examine a larger sample for potential asymmetry, we used a set of 23 healthy subjects studied on the Advance scanner. Although the spatial resolution $(\sim 7 \mathrm{~mm})$ of the 
Advance is less than half that of the HRRT ( $\sim 3 \mathrm{~mm})$, and reconstruction methods are different, the Advance scanner provided similar measures of asymmetry. Voxel volume was about $1.8 \mathrm{~mm}^{3}$ in HRRT images and about $8 \mathrm{~mm}^{3}$ in normalized Advance images.

\section{Image Analysis}

T1-weighed MR images were coregistered to averaged (0120 min) PET images using SPM2 (19) running on MATLAB (version 7.2.0.232; The MathWorks). Hippocampi and amygdalae were then manually delineated using PMOD (version 3.0; PMOD Technologies Ltd.) on axial coregistered T1-weighed MR images and regional time-activity curves extracted from the dynamic PET images. The hippocampal regions included cornua ammonis, dentate gyrus, and fimbriae. Anatomic criteria for delineating the hippocampi were adopted from published guidelines $(20,21)$, and accuracy was inspected using all 3 orthogonal projections. The volume of this region was about $2.9 \mathrm{~cm}^{3}$ in the affected hippocampus in patients with epilepsy and $3.4 \mathrm{~cm}^{3}$ in the unaffected hippocampus in patients with epilepsy and about 3.9 and $3.6 \mathrm{~cm}^{3}$ in the right and left hippocampus, respectively, in healthy subjects scanned with the HRRT camera. For regions outside the mesial temporal region, we used the Automated Anatomical Labeling template of volumes of interest (22) implemented in PMOD. For this analysis, PET images were spatially normalized using the ligand-specific normalization template. This template had been created using data PET images from 7 healthy subjects and normalization parameters derived from MR images. For healthy subjects from the Advance camera, we used the Automated Anatomical Labeling template for all brain regions. Data from the Automated Anatomical Labeling template were consolidated by volume-weighted averaging into the following volumes of interest: cerebellum, fusiform gyrus, occipital cortex, parahippocampal gyrus, parietal cortex, prefrontal cortex, lateral temporal cortex, striatum, and thalamus.

\section{Measurement of Brain Uptake}

As a primary evaluation, we measured decay-corrected brain uptake of radioactivity after injection of ${ }^{11} \mathrm{C}-\mathrm{PBR} 28$ from the hippocampus ipsilateral to the seizure focus, delineated on coregistered MR images, and compared it with that from the hippocampus contralateral to the seizure focus. To avoid confounding effects of delivery of radioligand into brain, we defined brain uptake as the average of standardized uptake values (SUVs) from 60 to $120 \mathrm{~min}$ (SUV $60-120)$. SUVs were calculated as radioactivity concentration divided by injected dose per body mass.

Small foci of higher uptake appeared on the superior border of the anterior hippocampus (see the "Results" section). These foci likely represented choroid plexus in the temporal horn of the lateral ventricle. Because choroid plexus in this region is difficult to separate from the hippocampus, we extracted this structure from PET images as follows. We used an automated algorithm to extract 50 contiguous voxels within a $7 \times 7 \times 7$ voxel cube centered on the hottest voxel within the area containing hippocampus and choroid plexus, from a PET image that had been averaged from 10 to $100 \mathrm{~min}$ and smoothed with a 3-mm gaussian kernel (full width at half maximum). Time-activity curves within this 50-voxel cluster were then calculated from the dynamic PET images. Finally, time-activity curves for the hippocampus were calculated by removing the area within the cluster from the dynamic image and then applying the region of interest for the whole hippocampus. The 50-voxel cutoff was selected on the basis of visual inspection.

\section{Statistical Analysis}

Data were analyzed with SPSS (release 17.0.0 [SPSS Inc.] for Windows [Microsoft]). Brain uptake from ipsilateral and contralateral hemispheres was compared using 2-tailed paired-samples $t$ tests, specifically testing the hypothesis of increased uptake ipsilateral to the seizure focus. Similarly, the paired-samples $t$ test was used in the healthy subjects to test the hypothesis of physiologically lateralized uptake of ${ }^{11} \mathrm{C}$-PBR28. In addition, we calculated an absolute asymmetry index for each subject as $200 \times \mid$ (left right $) /($ left + right $) \mid$ and compared these indices between the groups using ANOVA and an independent-samples $t$ test. To maintain a hypothesiswise type I error rate of 0.05 , observed $P$ values were corrected for multiple comparisons using the false discovery rate correction (23).

\section{RESULTS}

After injection of ${ }^{11} \mathrm{C}-\mathrm{PBR} 28$, a focal area of increased uptake was found in the hippocampal region in both patients and healthy subjects. Uptake in this focus was about $60 \%$ higher than that in the hippocampus. This focus occurred in both hemispheres and was anatomically located near the anterior and dorsal hippocampus (Fig. 1). On the basis of our prior imaging studies with ${ }^{11}$ C-PBR28 in monkeys (24), this focus was likely the choroid plexus in the temporal horn of the lateral ventricle, adjacent to the hippocampus. We confirmed the anatomic identity of the focus of high uptake by examining PET images from 1 healthy subject who, in addition to ${ }^{11} \mathrm{C}-\mathrm{PBR} 28$, had previously been scanned with ${ }^{11} \mathrm{C}$ - $\mathrm{N}$-desmethyl-loperamide $\left({ }^{11} \mathrm{C}\right.$-dLop) $(25) .{ }^{11} \mathrm{C}$-dLop is

FIGURE 1. Coronal sections of MR, PET, and fused PET/MR images demonstrate distribution of radioactivity in brain after injection of ${ }^{11} \mathrm{C}-\mathrm{PBR} 28$ in patient with left-sided temporal lobe epilepsy. PET image is summed from 0 to $120 \mathrm{~min}$. Right side of brain appears on left side of image. Area within red rectangles appears magnified in bottom row. There is higher uptake in ipsilateral than in contralateral side in choroid plexus (red arrow) and hippocampus (black arrow).

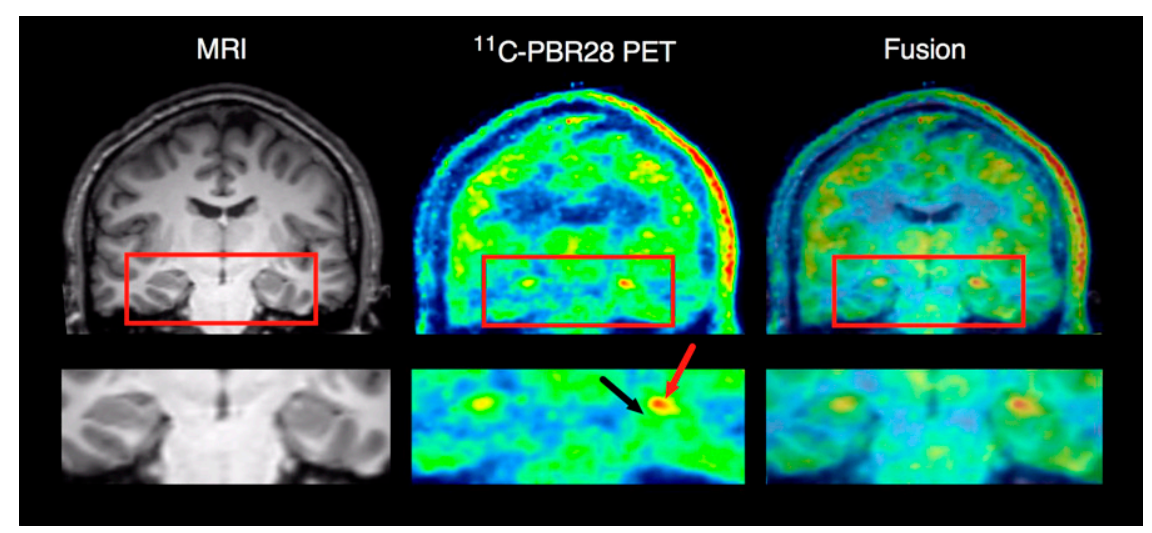


a substrate radioligand for P-glycoprotein, or P-gp, which is one of the major transporter molecules at the blood-brain barrier. In normal-brain parenchyma, P-gp blocks the entry of ${ }^{11} \mathrm{C}$-dLop, but for unknown reasons, ${ }^{11} \mathrm{C}$-dLop is avidly taken up in the choroid plexus (25). In this subject who underwent both ${ }^{11} \mathrm{C}-\mathrm{PBR} 28$ and ${ }^{11} \mathrm{C}$-dLop imaging, we noted spatial correspondence between the foci of high uptake in both images (Supplemental Fig. 1; supplemental materials are available online only at http://jnm.snmjournals.org). Therefore, the foci of high ${ }^{11} \mathrm{C}-\mathrm{PBR} 28$ uptake likely represent choroid plexus.

In patients, brain uptake was higher in the hippocampus, parahippocampal gyrus, amygdala, fusiform gyrus, and choroid plexus ipsilateral to seizure foci (Table 2; Fig. 2). The increased uptake in the hippocampus ipsilateral to the epileptic focus was more pronounced among patients with $(13 \% \pm 6 \%, n=9)$ than without $(5 \% \pm 4 \%, n=7)$ hippocampal sclerosis (2-tailed $P=0.009$ ) but did not correlate with duration of illness or frequency of seizures. Notably, asymmetry was significant even within patients without hippocampal sclerosis alone $(P=0.020, n=7)$.

We wondered if increased hippocampal uptake ipsilateral to seizure foci in patients was confounded by physiologic asymmetry in brain uptake of ${ }^{11} \mathrm{C}-\mathrm{PBR} 28$. No such asymmetry was seen among healthy subjects scanned with the HRRT camera, either in the hippocampus (right $0.1 \%$ larger than left; $P=0.855$ ) or choroid plexus (right $3.8 \%$ smaller than left; $P=0.163$ ). However, in the 23 healthy subjects scanned with the Advance camera, uptake was slightly higher in the left hemisphere than in the right hemisphere for the hippocampus $(1.9 \%, P<0.01)$ and fusiform gyrus $(1.5 \%, P=0.02)$ but not for the parahippocampal gyrus or amygdala. Although statistically significant, the asymmetry was substantially smaller in healthy subjects (range, $-10 \%$ to $11 \%$ ) than in patients (range, $-2 \%$ to $26 \%$ ). Finally, we compared absolute asymmetry indices between patients with epilepsy and healthy subjects scanned with the 2 PET cameras. ANOVA showed a significant difference between the 3 groups $\left(F_{2,43}=11.6, P<0.001\right)$. Posthoc contrast showed that patients with epilepsy differed from all healthy subjects $(\mathrm{t}=3.49, P=0.001)$ and that healthy subjects scanned with the HRRT camera did not differ from those scanned with the Advance camera $(P=0.063)$. The asymmetry index was $9.4 \pm 5.4$ in patients with epilepsy $(n=16)$ and $3.9 \pm 3.3$ in healthy subjects $(n=30)$. This analysis confirmed that the difference between affected and unaffected hippocampus in patients with epilepsy was significantly greater than the difference between the right and left hippocampus in healthy subjects.

The $95 \%$ confidence interval for the absolute asymmetry index in the 30 healthy subjects was 2.7-5.1. Four patients of 16 (patients 1, 3, 9, and 14; Table 1) had asymmetry values within the $95 \%$ confidence interval of healthy subjects. None of the subjects had hippocampal sclerosis. However, we caution against using lack of significant asymmetry as evidence for lack of inflammation, because increased uptake in the contralateral hemisphere could diminish asymmetry.

Could radioactivity from choroid plexus have contaminated the signal measured from adjacent regions via partialvolume effects? We simulated the spillover based on the spatial resolution of the camera and the distance between choroid plexus and amygdala. We assumed that the images had a resolution of $2.5 \mathrm{~mm}$ in full width at half maximum; that the choroid plexus and amygdala were 8 and $12 \mathrm{~mm}$ in diameter, respectively; that the choroid plexus and amygdala were separated by $2 \mathrm{~mm}$; and that the $\mathrm{SUV}_{60-120}$ was 1.8 in choroid plexus (measured from the affected hemi-

TABLE 2

Uptake of Radioactivity After Injection of ${ }^{11} \mathrm{C}-\mathrm{PBR} 28$ in Brain Regions Ipsilateral and Contralateral to Seizure Focus

\begin{tabular}{|c|c|c|c|c|c|}
\hline \multirow[b]{2}{*}{ Brain region } & \multicolumn{2}{|c|}{ Brain uptake $\left(\mathrm{SUV}_{60-120}\right)$} & \multirow[b]{2}{*}{ Difference } & \multirow[b]{2}{*}{ Effect size } & \multirow[b]{2}{*}{$P$} \\
\hline & Ipsilateral & Contralateral & & & \\
\hline Hippocampus & $1.15 \pm 0.4$ & $1.04 \pm 0.4$ & $+10 \%$ & 0.29 & $0.00007^{\star}$ \\
\hline Parahippocampal gyrus & $1.02 \pm 0.4$ & $0.94 \pm 0.3$ & $+8 \%$ & 0.23 & $0.0007^{\star}$ \\
\hline Amygdala & $1.16 \pm 0.4$ & $1.09 \pm 0.4$ & $+8 \%$ & 0.18 & $0.002^{*}$ \\
\hline Fusiform gyrus & $1.01 \pm 0.5$ & $0.97 \pm 0.4$ & $+4 \%$ & 0.08 & $0.002^{*}$ \\
\hline Choroid plexus & $1.81 \pm 0.7$ & $1.58 \pm 0.6$ & $+16 \%$ & 0.36 & $0.016^{*}$ \\
\hline Occipital cortex & $1.11 \pm 0.5$ & $1.09 \pm 0.5$ & $+1 \%$ & 0.04 & 0.190 \\
\hline Striatum & $0.87 \pm 0.4$ & $0.85 \pm 0.4$ & $+2 \%$ & 0.05 & 0.227 \\
\hline Thalamus & $1.13 \pm 0.5$ & $1.12 \pm 0.5$ & $+1 \%$ & 0.03 & 0.373 \\
\hline Lateral temporal cortex & $1.05 \pm 0.5$ & $1.03 \pm 0.4$ & $+1 \%$ & 0.04 & 0.441 \\
\hline Parietal cortex & $0.95 \pm 0.4$ & $0.94 \pm 0.4$ & $+1 \%$ & 0.03 & 0.539 \\
\hline Prefrontal cortex & $0.98 \pm 0.4$ & $0.99 \pm 0.4$ & $-1 \%$ & -0.02 & 0.545 \\
\hline Cerebellum & $1.41 \pm 0.6$ & $1.42 \pm 0.5$ & $-1 \%$ & -0.02 & 0.951 \\
\hline \multicolumn{6}{|c|}{$\begin{array}{l}\text { *Statistically significant, and survives false discovery rate correction for multiple comparisons. } \\
\text { Hippocampal values do not include contributions from choroid plexus, which was subtracted from total hippocampal volume using } \\
\text { automated clustering algorithm (see "Results" section). } P \text { values are uncorrected from } 2 \text {-tailed paired-samples } t \text { test. Brain uptake values } \\
\text { are mean } \pm S D \text {. }\end{array}$} \\
\hline
\end{tabular}


Hippocampus

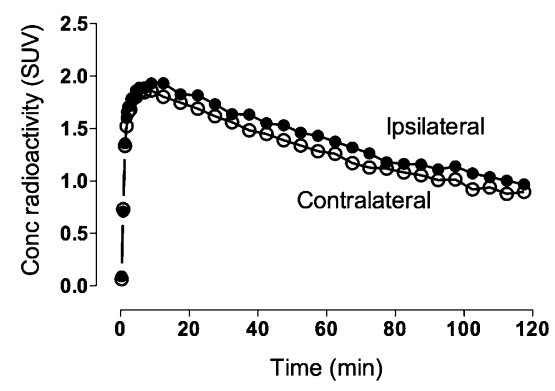

Parahippocampal gyrus

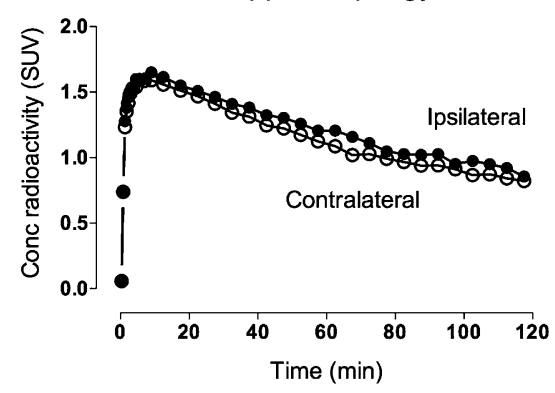

Amygdala

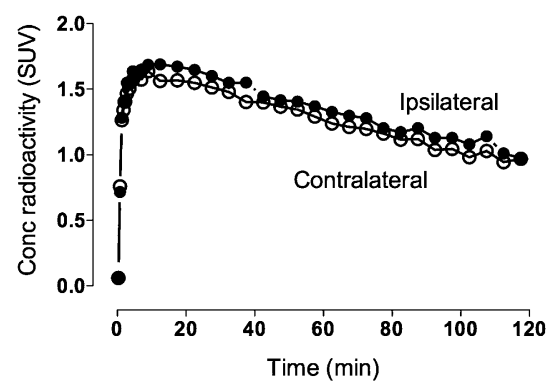

Fusiform gyrus

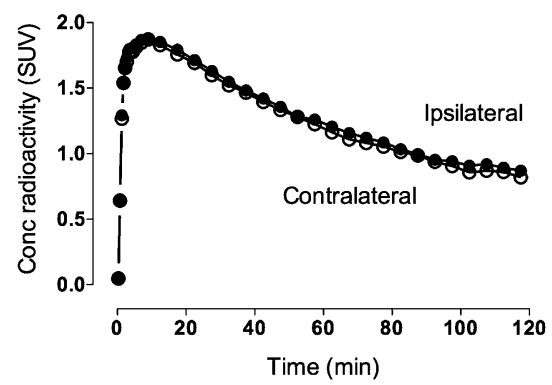

FIGURE 2. Uptake of radioactivity after injection of ${ }^{11} \mathrm{C}$-PBR28 ipsilateral and contralateral to seizure focus in brain regions that showed significant asymmetry. Values are mean of all 16 patients.
Choroid plexus

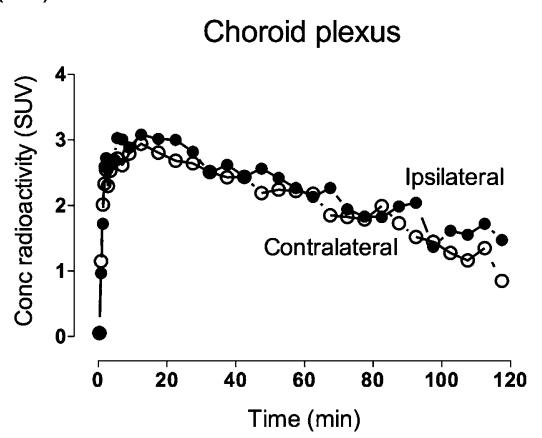

approach had the disadvantage of not being able to detect alterations that may be bilateral. For example, we clearly found increased TSPO binding in the ipsilateral, compared with the contralateral, hemisphere, but the contralateral hemisphere itself may have elevated binding, compared with that in control subjects. For such an analysis, we would need arterial blood data to measure the absolute binding (i.e., distribution volume) in each hemisphere of both patients and control subjects. Brain uptake values are dependent on how much tracer is available in plasma, and both the metabolism and the plasma protein binding may be different in medicated patients, compared with healthy subjects. Fully quantitative analysis of ${ }^{11} \mathrm{C}-\mathrm{PBR} 28$ binding requires arterial catheterization and will be done in the future to determine whether patients with epilepsy have increased TSPO binding only in the hemisphere containing the epileptogenic focus or in both hemispheres.

In contrast to our results, 1 previous study using ${ }^{11} \mathrm{C}-(R)$ PK11195 did not find a lateralized increase of binding in 3 patients with temporal lobe epilepsy (13). This negative result might have been caused by a small sample size; due to the fact that the patients were seizure-free on stable doses of antiepileptic medications, rather than being evaluated for 
uncontrolled epilepsy; or because of the low ratio of specific to nonspecific binding of ${ }^{11} \mathrm{C}-(R)$-PK11195.

Even with our improved PET ligand, ${ }^{11} \mathrm{C}-\mathrm{PBR} 28$, the increase was small, compared with decreased activity reported in PET studies of glucose metabolism (26). However, our results are comparable to increases in opiate receptor binding in temporal lobe epilepsy (27). Furthermore, the TSPO may be increased in only part of the hippocampus. In temporal lobe epilepsy, TSPO expression in vitro is most increased in the CA1 subfield of the hippocampus, which also shows the greatest atrophy (11). Thus, partial-volume effects may have prevented us from detecting this pronounced but spatially restricted increase in TSPO expression in vivo with PET. Other explanations for the small asymmetry are increased uptake in the contralateral and ipsilateral hemispheres and the possibility that the epileptogenic focus in some patients with temporal lobe epilepsy may be less affected by inflammation than that in others.

In all subjects, we noted foci of higher uptake superior to the anterior part of the hippocampus. These foci likely represented the choroid plexus in the temporal horn of the lateral ventricle, because the choroid plexus is known to have a high density of TSPO in monkeys $(24,28)$. Limited spatial resolution of PET and MRI impeded us from anatomically separating the choroid plexus from the hippocampus. Nevertheless, we confirmed the identity of the focus by comparing the distribution of uptake after injection of ${ }^{11} \mathrm{C}$ PBR28 with that after injection of ${ }^{11} \mathrm{C}-\mathrm{dLop}$, a substrate radioligand for $\mathrm{P}$-gp that is avidly taken up by the choroid plexus. In a healthy subject who underwent both PET scans, the focus of high uptake in the ${ }^{11} \mathrm{C}-\mathrm{PBR} 28$ image corresponded spatially to that in the ${ }^{11} \mathrm{C}$-dLop image (Supplemental Fig. 1), confirming that the focus in both images represents choroid plexus.

We considered performing partial-volume correction but decided not to do so for several reasons. This correction is commonly performed in pathologic conditions in which both the density of the target and the volume of tissue are decreased. The purpose of partial-volume correction is to determine whether the loss of receptors is proportionate to or disproportionately greater than the loss of tissue. The value of partial-volume correction is uncertain, however, in conditions such as temporal lobe epilepsy in which the density of the target (TSPO) is increased but the volume of tissue is generally decreased. Furthermore, not all tissues in the epileptogenic region have decreased volume. The region with the highest uptake is the choroid plexus, and we do not know whether the volume of this tissue is increased or decreased in the epileptogenic area. Finally, if we had done partial-volume correction-and assuming loss of tissue in the areas in the epileptogenic region-increased TSPO binding in temporal lobe epilepsy would have been even greater after partial-volume correction.

Physiologic asymmetry in the hippocampus and the fusiform gyrus was noted among 23 healthy subjects scanned with ${ }^{11} \mathrm{C}-\mathrm{PBR} 28$ using another PET camera with lower spatial resolution (Advance), such that values from the left hemisphere were less than $2 \%$ higher than values from the right hemisphere. Our results are only minimally confounded by this normal asymmetry. First, asymmetry was higher among the patients (range, $-2 \%$ to $26 \%$ ) than among healthy subjects (range, $-10 \%$ to $11 \%$ ), and patients had significantly higher absolute asymmetry indices. Second, patients showed significant asymmetry in regions that were not asymmetric among healthy subjects, such as the amygdala and parahippocampal gyrus. Finally, 5 of 16 patients (31\%) had the epileptogenic focus in the right not in the left hemisphere, which counterbalances for physiologic asymmetry.

Our results are not likely confounded by changes in cerebral blood flow in patients with temporal lobe epilepsy. We measured brain uptake of radioactivity from 60 to 120 min to avoid the early phase of uptake in which the cerebral blood flow has the largest effect. In addition, patients with temporal lobe epilepsy have reduced interictal blood flow ipsilateral to the seizure focus (29), which would only diminish the uptake of radioactivity after injection of ${ }^{11} \mathrm{C}-\mathrm{PBR} 28$.

About 10 percent of the approximately 100 subjects we have studied to date show negligible binding of ${ }^{11} \mathrm{C}$ PBR28 to TSPO in the brain and periphery. PBR28 has a much lower affinity for TSPOs in this subpopulation than in the larger population-about 10-fold lower for binding to membranes of white blood cells (15) and 25fold lower for binding to human brain tissue (17). In addition to low-affinity binders, Owen et al. (17) identified another group of subjects ( $\sim 30 \%$ of their study sample) that had mixed affinity - that is, a mixture of high- and low-affinity binding (17). For the current study, we excluded 1 patient with low-affinity binding based on PET images of the brain and following the criteria of Kreisl et al. (15). We doubt that in vivo imaging with ${ }^{11} \mathrm{C}-\mathrm{PBR} 28$ can detect mixed-affinity binders, who may therefore be included in our study. Nevertheless, inclusion of such individuals would not bias the within-subject comparison of ipsilateral and contralateral hemispheres assuming the distribution of mixed-affinity binding is uniform throughout the brain.

\section{CONCLUSION}

We found increased uptake of radioactivity after injection of ${ }^{11} \mathrm{C}$-PBR28 ipsilateral to the seizure focus in patients with temporal lobe epilepsy, suggesting an increased expression of TSPO. Exploratory analysis indicated that the adjacent parahippocampal gyrus, amygdala, fusiform gyrus, and choroid plexus are similarly affected. Future studies in larger samples should confirm this initial observation, establish whether other brain regions are involved, and measure absolute binding values using fully quantitative analysis. TSPO imaging may have utility in studies of the pathophysiology and treatment of temporal lobe epilepsy. 


\section{DISCLOSURE STATEMENT}

The costs of publication of this article were defrayed in part by the payment of page charges. Therefore, and solely to indicate this fact, this article is hereby marked "advertisement" in accordance with 18 USC section 1734.

\section{ACKNOWLEDGMENTS}

We thank Desiree Ferraris Araneta and the PET Department in the Clinical Center for the successful completion of this research. We also thank Drs. Ruben Kuzniecky and Jacqueline French for referring patients. This work was supported by the intramural programs of the National Institute of Mental Health (project Z01-MH-002852-04) and the National Institute of Neurological Disorders and Stroke (project 1Z01-NS002236-34) and grants from the Academy of Finland, the Finnish Cultural Foundation, the Finnish Foundation for Alcohol Studies, the Finnish Medical Foundation, the Instrumentarium Foundation, the Jalmari and Rauha Ahokas Foundation, the Paulo Foundation, the Research Foundation of Orion Corporation, and the Yrjö Jahnsson Foundation. No other potential conflict of interest relevant to this article was reported.

\section{REFERENCES}

1. Vezzani A, Balosso S, Ravizza T. The role of cytokines in the pathophysiology of epilepsy. Brain Behav Immun. 2008;22:797-803.

2. Ravizza T, Gagliardi B, Noe F, Boer K, Aronica E, Vezzani A. Innate and adaptive immunity during epileptogenesis and spontaneous seizures: evidence from experimental models and human temporal lobe epilepsy. Neurobiol Dis. 2008;29:142-160.

3. Rijkers K, Majoie HJ, Hoogland G, Kenis G, De Baets M, Vles JS. The role of interleukin-1 in seizures and epilepsy: a critical review. Exp Neurol. 2009; 216:258-271.

4. Aronica E, Boer K, van Vliet EA, et al. Complement activation in experimental and human temporal lobe epilepsy. Neurobiol Dis. 2007;26:497-511.

5. Crespel A, Coubes P, Rousset MC, et al. Inflammatory reactions in human medial temporal lobe epilepsy with hippocampal sclerosis. Brain Res. 2002; 952:159-169.

6. Sheng JG, Boop FA, Mrak RE, Griffin WS. Increased neuronal beta-amyloid precursor protein expression in human temporal lobe epilepsy: association with interleukin-1 alpha immunoreactivity. J Neurochem. 1994;63:1872-1879.

7. Kauffman MA, Moron DG, Consalvo D, Bello R, Kochen S. Association study between interleukin 1 beta gene and epileptic disorders: a HuGe review and meta-analysis. Genet Med. 2008;10:83-88.

8. Chen MK, Guilarte TR. Translocator protein $18 \mathrm{kDa}$ (TSPO): molecular sensor of brain injury and repair. Pharmacol Ther. 2008;118:1-17.

9. Papadopoulos V, Baraldi M, Guilarte TR, et al. Translocator protein (18kDa): new nomenclature for the peripheral-type benzodiazepine receptor based on its structure and molecular function. Trends Pharmacol Sci. 2006;27:402-409.

10. Cosenza-Nashat M, Zhao ML, Suh HS, et al. Expression of the translocator protein of $18 \mathrm{kDa}$ by microglia, macrophages and astrocytes based on immuno- histochemical localization in abnormal human brain. Neuropathol Appl Neurobiol. 2009;35:306-328.

11. Johnson EW, de Lanerolle NC, Kim JH, et al. "Central" and "peripheral" benzodiazepine receptors: opposite changes in human epileptogenic tissue. Neurology. 1992;42:811-815

12. Sauvageau A, Desjardins P, Lozeva V, et al. Increased expression of "peripheraltype" benzodiazepine receptors in human temporal lobe epilepsy: implications for PET imaging of hippocampal sclerosis. Metab Brain Dis. 2002;17:3-11.

13. Banati RB, Goerres GW, Myers R, et al. $\left[{ }^{11} \mathrm{C}\right](R)-\mathrm{PK} 11195$ positron emission tomography imaging of activated microglia in vivo in Rasmussen's encephalitis. Neurology. 1999;53:2199-2203.

14. Kumar A, Chugani HT, Luat A, Asano E, Sood S. Epilepsy surgery in a case of encephalitis: use of ${ }^{11} \mathrm{C}-\mathrm{PK} 11195$ positron emission tomography. Pediatr Neurol. 2008;38:439-442.

15. Kreisl WC, Fujita M, Fujimura Y, et al. Comparison of [(11)C]-(R)-PK 11195 and $\left[{ }^{11} \mathrm{C}\right] \mathrm{PBR} 28$, two radioligands for translocator protein $(18 \mathrm{kDa})$ in human and monkey: Implications for positron emission tomographic imaging of this inflammation biomarker. Neuroimage. 2010;49:2924-2932.

16. Briard E, Zoghbi SS, Imaizumi M, et al. Synthesis and evaluation in monkey of two sensitive ${ }^{11} \mathrm{C}$-labeled aryloxyanilide ligands for imaging brain peripheral benzodiazepine receptors in vivo. J Med Chem. 2008;51:17-30.

17. Owen DR, Howell OW, Tang SP, et al. Two binding sites for $\left[{ }^{3} \mathrm{H}\right] \mathrm{PBR} 28$ in human brain: implications for TSPO PET imaging of neuroinflammation. J Cereb Blood Flow Metab. 2010;30:1608-1618.

18. Fujita M, Imaizumi M, Zoghbi SS, et al. Kinetic analysis in healthy humans of a novel positron emission tomography radioligand to image the peripheral benzodiazepine receptor, a potential biomarker for inflammation. Neuroimage. 2008;40:43-52.

19. Friston KJ, Holmes AP, Worsley KJ, Poline JP, Frith C, Frackowiak RSJ. Statistical parametric maps in functional imaging: a general linear approach. Hum Brain Mapp. 1994;2:189-210.

20. Pantel J, O'Leary DS, Cretsinger K, et al. A new method for the in vivo volumetric measurement of the human hippocampus with high neuroanatomical accuracy. Hippocampus. 2000;10:752-758.

21. Van Erp TG, Saleh PA, Rosso IM, et al. Contributions of genetic risk and fetal hypoxia to hippocampal volume in patients with schizophrenia or schizoaffective disorder, their unaffected siblings, and healthy unrelated volunteers. Am J Psychiatry. 2002;159:1514-1520.

22. Tzourio-Mazoyer N, Landeau B, Papathanassiou D, et al. Automated anatomical labeling of activations in SPM using a macroscopic anatomical parcellation of the MNI MRI single-subject brain. Neuroimage. 2002;15:273-289.

23. Benjamini Y, Hochberg Y. Controlling the false discovery rate: a practical and powerful approach to multiple testing. JR Stat Soc B. 1995;57:289-300.

24. Imaizumi M, Briard E, Zoghbi SS, et al. Kinetic evaluation in nonhuman primates of two new PET ligands for peripheral benzodiazepine receptors in brain. Synapse. 2007;61:595-605.

25. Seneca N, Zoghbi SS, Liow JS, et al. Human brain imaging and radiation dosimetry of ${ }^{11} \mathrm{C}-\mathrm{N}$-desmethyl-loperamide, a PET radiotracer to measure the function of P-glycoprotein. J Nucl Med. 2009;50:807-813.

26. Liew CJ, Lim Y-M, Bonwetsch R, et al. ${ }^{18} \mathrm{~F}-\mathrm{FCWAY}$ and ${ }^{18} \mathrm{~F}-\mathrm{FDG}$ PET in MRInegative temporal lobe epilepsy. Epilepsia. 2009;50:234-239.

27. Frost JJ, Mayberg HS, Fisher RS, et al. Mu-opiate receptors measured by positron emission tomography are increased in temporal lobe epilepsy. Ann Neurol. 1988;23:231-237.

28. Cymerman U, Pazos A, Palacios JM. Evidence for species differences in 'peripheral' benzodiazepine receptors: an autoradiographic study. Neurosci Lett. 1986;66:153-158.

29. Gaillard WD, Fazilat S, White $\mathrm{S}$, et al. Interictal metabolism and blood flow are uncoupled in temporal lobe cortex of patients with complex partial epilepsy. Neurology. 1995;45:1841-1847. 